\title{
Childhood antecedents of schizophrenia: Will understanding aetiopathogenesis result in schizophrenia prevention?
}

\author{
James G. Scott ${ }^{1,2,3}$
}

Received: 8 January 2016/Accepted: 17 January 2016/Published online: 29 January 2016

(C) Springer-Verlag Berlin Heidelberg 2016

Prevention is particularly important for those disorders whose course is chronic and disabling. Schizophrenia and other psychotic disorders have high levels of disability [1] and challenges with treatment non-adherence and the associated risk of iatrogenic harm from antipsychotic medication makes prevention an urgent imperative. Initial studies identifying adolescents and young adults at ultrahigh risk of psychosis were accompanied by optimism that transition to schizophrenia could be prevented [2]. However, pharmacological interventions in the prodromal phase have been of no benefit, cognitive behavioural therapy has shown limited benefits to date in preventing psychosis transition [3] and one study demonstrating effectiveness of omega-3-polyunsaturated fatty acids in preventing transition to psychosis [4] has yet to be replicated. The delivery of interventions to those adolescents and young adults in the prodromal phase of psychosis may be too late to prevent illness progression. It is well established that in some individuals, the antecedents for psychosis are evident from a young age, many years before the phenotypic expression of psychosis is evident [5].

Invited commentary on "Toward earlier identification and preventative intervention in schizophrenia: evidence from the London Child Health and Development Study".

James G. Scott

james.scott@health.qld.gov.au

1 Discipline of Psychiatry, School of Medicine, The University of Queensland, UQ Centre for Clinical Research, Herston, QLD 4029, Australia

2 Queensland Centre for Mental Health Research, The Park Centre for Mental Health, Wacol, QLD 4076, Australia

3 Metro North Mental Health, Royal Brisbane and Women's Hospital, Herston, QLD 4029, Australia
The London Child Health and Development Study (CHAD) has attempted to address this by examining the developmental course towards schizophrenia in pre and peri-pubertal children. In this issue, Laurens and Cullen summarise the methodology and key findings of CHAD to date [6]. Baseline screening for antecedents of schizophrenia was conducted in a large community sample $(n=7966)$ of children aged 9-12 years. Antecedents comprised of a triad of signs and symptoms; (1) childreported psychotic-like experiences (PLEs), (2) child-reported emotional symptoms and/or caregiver-reported behavioural or social problems, and (3) caregiver-reported abnormalities in speech and/or motor development. These three antecedents were reported in almost $10 \%$ of the sample. A sub-sample $(n=111)$ comprising of typically developing children $(n=46)$, those with a family history of schizophrenia $(n=26)$, those with antecedents of schizophrenia $(n=33)$ and those with both family history and antecedents $(n=6)$ have been investigated longitudinally. Psychopathological, neurobiological and cognitive markers have been examined biennially in these children as they grow through the developmental stage where schizophrenia typically emerges [6].

The participants of CHAD are in the early stages of the high risk period for developing schizophrenia and it will be a further decade before they pass through early adulthood when schizophrenia typically occurs. At this time, the predictive power of phenotypic, cognitive and neurobiological markers in these young children may be ascertained. Already, those children with antecedents of schizophrenia have been shown to have psychopathological signs, and neurocognitive and neuroimaging changes many of which share commonalities with adults who have schizophrenia and are able to be differentiated from typically developing children [6]. 
In spite of the many strengths of CHAD, the small numbers and likely insufficient power may preclude accurate determination of those children most at risk for future schizophrenia. However, the study provides an excellent protocol for future research, the methodology will likely be replicated with larger numbers and novel information regarding potential antecedents for schizophrenia will be identified.

The heterogeneity of the schizophrenia syndrome is a challenge facing all aetiopathological research. Whilst some with schizophrenia have an atypical neurodevelopmental course, others show no evidence of childhood antecedent signs and symptoms. Those identified by atypical neurodevelopment represent only a sub-sample of people with schizophrenia, a broad syndrome currently defined by symptoms rather than underlying aetiopathological processes. Studies like CHAD may assist in redefining the diagnostic boundaries of the psychosis syndromes.

CHAD also has an intervention arm for those with PLEs aimed at reducing distress and motivating help seeking by parents [6]. A selective intervention of cognitive behavioural therapy years before the likely onset of schizophrenia has been shown to be acceptable in a case series of four children with PLEs and emotional distress. However, the feasibility of such an intervention needs demonstrating in a larger sample. High levels of participant and parent involvement required as per this case series where children attended between six and 15 sessions will prove a barrier to the widespread implementation of any selective preventative intervention. The reported intervention is better considered an intensive targeted intervention for highly symptomatic children who have co-occurring PLEs. A randomised controlled trial assessing effectiveness is underway and the results will inform treatment for these children. To date, there are few if any other studies reporting interventions for PLEs in this young age group.

Schizophrenia shares risk factors common to most other mental disorders and universal interventions that improve the mental health of the population may assist in preventing psychosis. Addressing parental mental illness in the postpartum period, reducing family violence, improving parenting skills and reducing childhood maltreatment and bullying have the greatest potential to reduce the prevalence of emotional and behavioural disorders in children and adolescents [7]. Whilst the short-term benefits of interventions addressing these risk factors has been demonstrated, evidence of long-term efficacy or cost effectiveness has yet to be established.

There are many challenges in supporting parents to obtain mental health care for their children. Parents of children and adolescents with emotional and behavioural problems underutilise mental health services because of financial and practical barriers such as long wait times to access help, transport and child care costs for siblings [8]. Selective interventions for children with antecedents of schizophrenia may be more feasibly delivered by other methods such as internet-based platforms [9] which are more accessible and affordable for parents and their children. In spite of the improved understanding of risk factors and antecedents for mental disorders, there has been no reduction in their prevalence in children and adolescents [10] and innovative ways of providing universal and selective preventative interventions are required.

The London Child Health and Development Study has made a novel, meaningful contribution to understanding the antecedents of schizophrenia. As participants grow through their adult years and transition through the period of risk for psychosis, biomarkers predictive of schizophrenia may be identified. It remains unclear if better identification of children at risk of schizophrenia will translate to parents taking steps to intervene so as to reduce the onset of future psychosis. Policy makers need to embrace novel approaches that incentivise parents and society more broadly to improve the mental health of children thereby reducing the prevalence and associated disability of schizophrenia and other persistent mental disorders.

Acknowledgments JGS is supported by a National Health and Medical Research Council Practitioner Fellowship Grant APP1105807.

\section{Compliance with ethical standards}

Conflict of interest There are no conflicts of interest.

\section{References}

1. Morgan C, Lappin J, Heslin M, Donoghue K, Lomas B, Reininghaus $\mathrm{U}$, Onyejiaka A, Croudace T, Jones PB, Murray RM, Fearon P, Doody GA, Dazzan P (2014) Reappraising the longterm course and outcome of psychotic disorders: the AESOP-10 study. Psychol Med 44(13):2713-2726. doi:10.1017/ s0033291714000282

2. Yung AR, Phillips LJ, Yuen HP, Francey SM, McFarlane CA, Hallgren M, McGorry PD (2003) Psychosis prediction: 12-month follow up of a high-risk ("prodromal") group. Schizophr Res 60(1):21-32

3. Stafford MR, Jackson H, Mayo-Wilson E, Morrison AP, Kendall T (2013) Early interventions to prevent psychosis: systematic review and meta-analysis. BMJ (Clin Res Ed) 346:f185. doi:10. 1136/bmj.f185

4. Amminger GP, Schafer MR, Papageorgiou K, Klier CM, Cotton SM, Harrigan SM, Mackinnon A, McGorry PD, Berger GE (2010) Long-chain omega-3 fatty acids for indicated prevention of psychotic disorders: a randomized, placebo-controlled trial. Arch Gen Psychiatry 67(2):146-154. doi:10.1001/arch genpsychiatry.2009.192

5. Welham J, Isohanni M, Jones P, McGrath J (2009) The antecedents of schizophrenia: a review of birth cohort studies. Schizophr Bull 35(3):603-623. doi:10.1093/schbul/sbn084 
6. Laurens KR, Cullen AE (2015) Toward earlier identification and preventative intervention in schizophrenia: evidence from the London Child Health and Development Study. Soc Psychiatry Psychiatr Epidemiol. doi:10.1007/s00127-015-1161-8015-1161-8

7. Patel V, Chisholm D, Parikh R, Charlson FJ, Degenhardt L, Dua T, Ferrari AJ, Hyman S, Laxminarayan R, Levin C, Lund C, Medina Mora ME, Petersen I, Scott J, Shidhaye R, Vijayakumar L, Thornicroft G, Whiteford H (2015) Addressing the burden of mental, neurological, and substance use disorders: key messages from Disease Control Priorities, 3rd edition. Lancet (London, England). doi:10.1016/s0140-6736(15)00390-6

8. Iskra W, Deane FP, Wahlin T, Davis EL (2015) Parental perceptions of barriers to mental health services for young people. Early Interv Psychiatry. doi:10.1111/eip.12281
9. Manicavasagar V, Horswood D, Burckhardt R, Lum A, HadziPavlovic D, Parker G (2014) Feasibility and effectiveness of a web-based positive psychology program for youth mental health: randomized controlled trial. J Med Internet Res 16(6):e140. doi:10.2196/jmir.3176

10. Bor W, Dean AJ, Najman J, Hayatbakhsh R (2014) Are child and adolescent mental health problems increasing in the 21st century? A systematic review. Aust N Z J Psychiatry 48(7):606-616. doi: $10.1177 / 0004867414533834$ 